\title{
The significance of the Hansen Ideal space frame
}

\author{
E.F.M. Jochim ${ }^{\star}$
}

German Aerospace Centre, Oberpfaffenhofen, D-82234 Wessling, Germany

Received 2011 Sep 2, accepted 2012 Jun 7

Published online 2012 Oct 2

Key words celestial mechanics - history and philosophy of astronomy - methods: analytical - reference systems

Known and unknown properties of Hansen Ideal coordinates are summarized. It is shown that the ideal space frame is a general and necessary component of basic celestial mechanics and astrodynamics, as well as of any theory of motion. A typical consequence is the intimate correlation of the Hansen frame with the Lagrange constraint within the method of the variation of the parameters. The use of observations in the ideal frame may allow conclusions on the intergalactic fundamental coordinate system.

(c) 2012 WILEY-VCH Verlag GmbH \& Co. KGaA, Weinheim

\section{Table of symbols}

$A_{k}^{(\nu)}$ $b_{\mathrm{R}}, b_{\mathrm{T}}, b_{\mathrm{N}}$

$D_{p}$

$\boldsymbol{D}_{q^{(\mathrm{L})}}$

$\boldsymbol{D}_{q^{(\mathrm{I})}}$

G

$\boldsymbol{d}_{0}, \boldsymbol{f}_{0}, \boldsymbol{c}_{0}$

$\boldsymbol{k}_{1}, \boldsymbol{k}_{2}, \boldsymbol{c}_{0}$ $i$

$\boldsymbol{p}_{i}$

$p_{i j}$
$\boldsymbol{q}_{j}^{(\mathrm{I})}$

$\boldsymbol{q}_{j}^{(\mathrm{I})}$

$q_{i j}$

$\boldsymbol{r}, \dot{r}$

$\dot{\boldsymbol{r}}_{q}$ $q_{j}^{k} \boldsymbol{q}_{k}^{(\mathrm{I})}$
Parameters of a curve presented in polar equation ( $k=1,2,3 \ldots$ - number of parameter, $(\nu)$ - kind of curve). In unperturbed case: $A_{k}^{(\nu)}=$ const.

Accelerations in radial, transversal, normal direction $\left[\mathrm{km} / \mathrm{s}^{2}\right]$

System proper motion vector of $\boldsymbol{p}_{i^{-}}$ system: $\boldsymbol{D}_{p i}=D_{p}^{i} \boldsymbol{p}_{i}$

System proper motion vector of the Leibniz-system

System proper motion vector of the $\boldsymbol{q}_{j}^{(\mathrm{I})}$ system (Hansen system): $\boldsymbol{D}_{q^{(\mathrm{I})}}=D_{q}^{j} \boldsymbol{q}_{j}^{(\mathrm{I})}$ Equal area parameter $\left[\mathrm{km}^{2} / \mathrm{s}\right]$

Basic vectors of the apsidal system

Basic vectors of the nodal system

Inclination [deg]

Basis vectors of the inertial system (Newton frame) $(i=1,2,3)$

Components of $\dot{\boldsymbol{p}}_{i}$-vectors: $\dot{\boldsymbol{p}}_{i}=p_{i}^{j} \boldsymbol{p}_{j}$

Basis vectors of the ideal system (Hansen frame) $(j=1,2,3)$

Components of $\dot{\boldsymbol{q}}_{j}^{(\mathrm{I})}$-vectors: $\dot{\boldsymbol{q}}_{j}^{(\mathrm{I})}=$

State vector: direction vector, absolute velocity vector

Relative velocity vector with respect to the $\boldsymbol{q}_{j}^{(\mathrm{I})}$-system: $\dot{\boldsymbol{r}}_{q}=\dot{y}^{j} \boldsymbol{q}_{j}^{(\mathrm{I})}$ $\boldsymbol{r}_{0}, \boldsymbol{q}_{0}, \boldsymbol{c}_{0}$

is vectors of co-moving orbit system (Leibniz frame), $\boldsymbol{r}_{0}$ in radial, $\boldsymbol{q}_{0}$ in transversal ("transradial"), $\boldsymbol{c}_{0}$ in normal direction

$r, r_{\mathrm{P}} \quad$ Radius, radius of pericentre distance [km]

$t, t_{\mathrm{P}} \quad$ Time, time of pericentre passage [s]

$u \quad$ Argument of latitude [rad]

$V \quad$ Velocity $[\mathrm{km} / \mathrm{s}]$

$v \quad$ True anomaly [rad]

$x_{i}, \dot{x}_{i} \quad$ Cartesian coordinates in the $\boldsymbol{p}_{i}$-system

$y_{j}, \dot{y}_{j} \quad$ Cartesian coordinates in the $\boldsymbol{q}_{j}^{(\mathrm{I})}$ system

$\delta_{i j} \quad$ Kronecker tensor

$\zeta \quad$ Orbit angle (first Hansen angle) [rad]

$\dot{\zeta} \quad$ Absolute variation of orbit angle $[\mathrm{rad} / \mathrm{s}]$ as measured from proper motion of radial direction vector $\left(\dot{\zeta}=G / r^{2}=\right.$ $\left.\left|\dot{\boldsymbol{r}}_{0}\right|\right)$

$\dot{\zeta}_{\mathrm{B}} \quad$ Variation of orbit angle with respect to a basic system as computed from orbit determination $[\mathrm{rad} / \mathrm{s}]$

$\dot{\zeta}_{\mathrm{P}} \quad$ Variation of orbit angle due to variation of basic system with respect to inertial fundamental system [ $\mathrm{rad} / \mathrm{s}]$

$\eta \quad$ Spatial rotation angle (second Hansen angle) [rad]

$\mu \quad$ Centric gravitational constant $\left[\mathrm{km}^{3} / \mathrm{s}^{2}\right]$

$\sigma \quad$ Longitude in orbit of ascending node [rad] (related to departure point of the Hansensystem)

$\psi \quad$ General orbit angle [rad] for any osculating orbit related system

$\Omega \quad$ Right ascension (or ecliptical longitude) of ascending node [rad]

Argument of pericenter (perihel, perigee $)[\mathrm{rad}](\omega=\zeta-\sigma)$

\footnotetext{
^ Corresponding author: fritz.jochim@dlr.de
} 


\section{Introduction}

Peter Andreas Hansen (Tondern in Slesvig 1795-Gotha 1874) introduced the term "ideal coordinates" into celestial mechanics. He stated (Hansen 1857, p.66): "I call Ideal Coordinates of a planet, of a comet or of a satellite, all those coordinates of these objects, when they have the property that not only these coordinates but also their derivatives with respect to time have the same form in the perturbed and in the unperturbed case." By this definition, not only "ideal coordinates" but also any other property is introduced as being an "ideal" parameter if it is of identical form in a "perturbed" and "unperturbed" case. Use of this behaviour was made by Hansen himself in developing his perturbation theory for minor planets as well as for his renowned investigation of lunar motion (cf. Hansen 1864). This theory uses a separation into the investigation of a fictitious body moving on an unperturbed conic section (in planetary theory always an ellipse) and the deviation of a true body moving under any acceleration from this unperturbed motion. Among others (like G. W. Hill in 1881), Brown (1896) has extended the Hansen theory to his investigation of lunar motion. Extensions of Hansen's developments will be found in the work by $\mathrm{H}$. Gylden and his pupil M. Brendel (as cited in Tisserand 1896, Tome IV, p.376 ff), but without insight into the general behaviour of this detection. Tisserand has prepared an intensive and general introduction to Hansen's perturbation theory. A similar presentation will be found in Stumpff (1974, Volume III, pp. 163-229).

Musen (1959) and Herget (1959) (see also Brouwer $1959 \mathrm{~b})$ adopted the method for initial investigations into the motion of artificial Earth satellites, introducing the inclusion of accelerations due to any gravitational term. The basic idea of "ideal coordinates" was not affected by all these applications of Hansen's ideas. In some papers, Deprit (1975, 1976) investigated the use of ideal coordinates with respect to special selections of "ideal frames" and the use of "ideal elements". Applications of the Hansen ideal coordinates for satellite orbit computations will be found in many papers since launch of the first satellite (see, e.g., Musen 1958; Herget \& Musen 1958; Musen 1959, 1961; Yarov-Yarovoi 1961; Musen 1963, 1968; Phelan 1962; Fisher 1963; Vinh 1969; Palacios \& Calvo 1996; Palacios 2000; etc.). In an implicit manner, Van der Ha (1985) developed orbit variational equations using a kind of orbit angle similar to Hansen's orbit angle, however with no reference to the Hansen theory.

The advantage of the Hansen Ideal systems as found by Hansen himself is the clear separation of in-plane and out of plane perturbations, as well as the use of the orbit angle related to the departure point of the Hansen system. In the previous papers an improvement in the numerical integration of the satellite equations of motion by use of Ideal Coordinates is attempted. However, the theoretical basis of the Hansen Ideal systems is accepted as known before. No attempt will be found in these papers in order to achieve a deeper and wider theoretical understanding of these systems.
1. How did Hansen detect ideal coordinates?

2. Did he accidentally detect ideal coordinates while applying the method of Eulerian variation of the constants or was the detection a necessary conclusion which was lost during the application of the ideal coordinates in his perturbation theory?

3. Do ideal coordinates play the only role in the context of the special perturbation theory within the family of Hansen perturbation theories or do they have their place in the core of any general theory of motion?

"Ideal coordinates" are known in literature under different names. Brumberg (1995), for example, uses the term "Hansen coordinates". In the present paper, we use the term "Hansen Idealcoordinates". In future, a meaningful terminology should include the name Hansen in order to respect the central role of these coordinates: Hansen coordinates, or Hansen system, or Hansen frame.

\section{General properties of the Hansen Ideal coordinate systems}

In this section some general properties of Hansen Ideal coordinates will be collected in the frame of classical orbital mechanics. All investigations will be treated in an Euclidean space frame. Let $\boldsymbol{p}_{i}$ be the orthonormal basis of a fixed space frame $\left(\boldsymbol{p}_{i} \cdot \boldsymbol{p}_{j}=\delta_{i j}\right)^{1}$, then the radius and the velocity vector of a celestial body with Cartesian coordinates $x^{i}$ ( $i$ as index, $i=1,2,3$ ) are represented by

$\boldsymbol{r}=x^{i} \boldsymbol{p}_{i}, \quad \dot{\boldsymbol{r}}=\dot{x}^{i} \boldsymbol{p}_{i}+x^{i} \dot{\boldsymbol{p}}_{i}$.

The velocity vector $\dot{\boldsymbol{r}}$ includes all motions of the basic system. It will be called the absolute velocity vector. Correspondingly, the relative velocity vector with respect to the $\boldsymbol{p}_{i}$-system will be defined by

$\dot{\boldsymbol{r}}_{p}:=\dot{x}^{i} \boldsymbol{p}_{i}$.

The vectors $\dot{\boldsymbol{p}}_{i}$ describe the proper motion of the basic system which might be represented in the variational equations

$$
\begin{array}{ccc}
\dot{\boldsymbol{p}}_{1}= & p_{12} \boldsymbol{p}_{2}+p_{13} \boldsymbol{p}_{3}, \\
\dot{\boldsymbol{p}}_{2}= & -p_{12} \boldsymbol{p}_{1} & +p_{23} \boldsymbol{p}_{3}, \\
\dot{\boldsymbol{p}}_{3}= & -p_{13} \boldsymbol{p}_{1}-p_{23} \boldsymbol{p}_{2} .
\end{array}
$$

These equations might be called general Frenet equations according to the Frenet equations in the curvature theory in differential geometry. Correspondingly, a general Darboux vector of the $\boldsymbol{p}_{i}$-system can be defined by

$\boldsymbol{D}_{p}:=D_{p}^{i} \boldsymbol{p}_{i}, \quad \dot{\boldsymbol{p}}_{i}=\boldsymbol{D}_{p} \times \boldsymbol{p}_{i}$,

so the relations hold

$D_{p 1}=p_{23}=-p_{32}$,
$D_{p 2}=p_{31}=-p_{13}$,
$D_{p 3}=p_{12}=-p_{21}$.

This generalized Darboux vector might be called the "absolute system proper motion vector". It describes the

\footnotetext{
1 The dot indicates a scalar product, $\delta_{i j}$ the Kronecker symbol; the Einstein summation terminology is used in this paper.
} 
proper motion of the system with respect to the cosmic inertial frame. If the basic system does not show any proper motion besides that of a uniform rectilinear motion, it will be called "inertial". In this case

$\dot{\boldsymbol{p}}_{i} \equiv 0, \quad \boldsymbol{D}_{p} \equiv 0$,

and the absolute and the relative velocity vector will coincide.

Assuming a $\boldsymbol{q}_{j}$-space frame in the osculating orbital plane, defined by the orbit normal vector

$\boldsymbol{r} \times \dot{\boldsymbol{r}}=: \boldsymbol{c}$,

the radius and velocity vector with Cartesian coordinates $y^{j}$ $(j=1,2,3)$ are defined by

$\boldsymbol{r}=y^{j} \boldsymbol{q}_{j}, \quad \dot{\boldsymbol{r}}=\dot{y}^{j} \boldsymbol{q}_{j}+y^{j} \dot{\boldsymbol{q}}_{j}$.

This system is related to the motion of the celestial body. Therefore, in general, if "orbital perturbations" have to be included, not all $\dot{\boldsymbol{q}}_{j}$ will necessarily vanish. In the sense of Hansen, this system is called an "ideal system" if the presentation of the velocity is the same in the unperturbed $\left(\dot{\boldsymbol{q}}_{j} \equiv 0\right)$ as in the perturbed ( $\dot{\boldsymbol{q}}_{j} \neq 0$ ) motion case. This leads to

Theorem 1 (Hansen 1857): A coordinate system is Hansen Ideal if, and only if, for the velocity vector the condition

$y^{j} \dot{\boldsymbol{q}}_{j}=0$

holds.

This sentence does not include any answer on the existence of such an ideal system.

Remark: At this point it cannot be concluded why Hansen came to the idea of an ideal system. In his paper of 1857 , no hint is to be found to this question.

Because of the close relationship of the $\boldsymbol{q}_{j}$-coordinate system to the motion of a celestial body, Hansen proposes to set $y_{3} \equiv 0$. The basic plane of a Hansen Ideal frame is assumed to be the (osculating) orbital plane of the moving body. This is not necessary to understand ideal systems. However, it facilitates the investigation and, therefore, we follow this proposal. The definition of the relative velocity vector gives, as a necessary consequence of Theorem 1:

Theorem 2 (Hansen 1857): A coordinate system is Hansen Ideal if, and only if, the absolute velocity vector is always identical to the relative velocity vector

$\dot{\boldsymbol{r}}=\dot{\boldsymbol{r}}_{q}=\dot{y}^{i} \boldsymbol{q}_{j}$.

A first application of Hansen Ideal systems follows for any system proper motion vector. Let be $\boldsymbol{D}_{g}:=D_{g}^{i} \boldsymbol{g}_{i}$ the system proper motion vector with respect to any orthonormal basis $\boldsymbol{g}_{i}$. Its variation is

$\dot{\boldsymbol{D}}_{g}=\dot{D}_{g}^{i} \boldsymbol{g}_{i}+D_{g}^{i} \dot{\boldsymbol{g}}_{i}$.

Using formula (4), valid for the $\boldsymbol{g}_{i}$-system, it follows

$D_{g}^{i} \dot{\boldsymbol{g}}_{i}=D_{g}^{i} \boldsymbol{D}_{g} \times \boldsymbol{g}_{i}=\boldsymbol{D}_{g} \times \boldsymbol{D}_{g}=0$, $\dot{\boldsymbol{D}}_{g}=\dot{D}_{g}^{i} \boldsymbol{g}_{i}$.

Therefore, Theorem 2 is fulfilled. The variation of a system proper motion vector is invariant with respect to any proper motion of the basic system. In terms of Hansen 's definition: Theorem 3: The absolute system proper motion vector of any coordinate system forms with its basis a Hansen Ideal coordinate system.

This theorem also answers the search for Hansen Ideal systems: It demonstrates at least the existence of Hansen Ideal systems. In the sequel we use the notation $\boldsymbol{q}_{j}^{(\mathrm{I})}$-system for the Hansen Ideal frame.

A general property of Hansen Ideal coordinate systems with interesting applications is described in

Theorem 4 (Stumpff 1974, p.173): If a position vector $\boldsymbol{r}=$ $y^{j} \boldsymbol{q}_{j}^{(\mathrm{I})}$ forms a Hansen Ideal coordinate system with its basis $\boldsymbol{q}_{j}^{(\mathrm{I})}$, then the coordinates of any scalar field $f(\boldsymbol{r})$ are Hansen Ideal coordinates.

Proof: If

$\dot{\boldsymbol{r}}=\dot{y}^{j} \boldsymbol{q}_{j}^{(\mathrm{I})}, \quad y^{j} \dot{\boldsymbol{q}}_{j}^{(\mathrm{I})}=0$,

it follows

$$
\begin{aligned}
\dot{f} & =\frac{\mathrm{d} f(\boldsymbol{r})}{\mathrm{d} t}=\frac{\partial f}{\partial \boldsymbol{r}} \cdot \dot{\boldsymbol{r}}=\nabla f \cdot \dot{\boldsymbol{r}}= \\
& =\dot{x}^{i} \boldsymbol{p}_{i} \cdot \frac{\partial f}{\partial x^{j}} \boldsymbol{p}_{j}=\dot{x}^{i} \frac{\partial f}{\partial x^{i}} \quad \text { (q.e.d.). }
\end{aligned}
$$

A fascinating behaviour of Hansen Ideal coordinate systems was found by Hansen himself: From $y^{j} \dot{\boldsymbol{q}}_{j}^{(\mathrm{I})}=0$ and the system proper motion vector $D_{q^{(\mathrm{I})}}$ of a Hansen Ideal $\boldsymbol{q}_{j}^{(\mathrm{I})}$-system with $\dot{\boldsymbol{q}}_{j}^{(\mathrm{I})}=\boldsymbol{D}_{q^{(\mathrm{I})}} \times \boldsymbol{q}_{j}^{(\mathrm{I})}$ it follows $0=y^{j} \dot{\boldsymbol{q}}_{j}^{(\mathrm{I})}=\boldsymbol{D}_{q^{(\mathrm{I})}} \times \boldsymbol{r}$.

That means

$\boldsymbol{D}_{q^{(\mathrm{I})}} \times \boldsymbol{r}=0 \Leftrightarrow$ System is Hansen Ideal.

Theorem 5 (Hansen 1857): Any vector in a threedimensional linear vector space forms with its basis a Hansen Ideal coordinate system, if and only if its system proper motion vector always coincides with the direction of the vector.

The original formulation of Hansen (1857): "In each ideal coordinate system which is related to moving axes, the instantaneous rotational axis always coincides with the radius vector of the planet or comet or satellite."

A combination of Hansen Ideal coordinate systems and the proper motion of these systems is given by the following Theorem 6 (Stumpff 1974): A position vector $\boldsymbol{r}=y^{j} \boldsymbol{q}_{j}$ forms with its basis a Hansen Ideal system, if and only if the components of the variational vectors of this system obey the condition

$y^{j} q_{j}^{(k)}=0 \quad($ for $k=1,2,3)$.

Proof: A Hansen Ideal system requires (see Theorem 1) $y^{j} \dot{\boldsymbol{q}}_{j}=0$. The variation vector of the basic system is given by (cf. Eq. 4) $\dot{\boldsymbol{q}}_{j}=q_{j}^{k} \boldsymbol{q}_{k}$. Hence $y^{j} q_{j}^{k} \boldsymbol{q}_{k}=0$. Here the $\boldsymbol{q}_{k}$ form a basis of the vector space, therefore they are linearly independent and consequently $y^{j} q_{j}^{(k)}=0$ (q.e.d.).

Theorem 6 allows a statement on the number of possible Hansen Ideal coordinate systems. From Eq. (17) it follows $q_{23} y_{2}-q_{31} y_{1}=0$,

$q_{31} y_{3}-q_{12} y_{2}=0$,

$q_{12} y_{1}-q_{23} y_{3}=0$. 
This means, the condition of Hansen Ideal frames only allows the selection of two parameters whereas the third will be a free parameter. As a conclusion it follows

Theorem 7 (Stumpff 1974): For each motion of a moving body there may be constructed an infinite number of possible Hansen Ideal coordinate systems.

It is clear that Theorem 5 is identical with Theorem 7. From Eq. (18) it follows

$\frac{q_{23}}{y_{1}}=\frac{q_{31}}{y_{2}}=: A$,

therefore

$\boldsymbol{D}_{q}=A \boldsymbol{r}$

in the case of Hansen Ideal coordinate systems, which is the same as Eq. (16).

Further conclusions are

Theorem 8: The core property of a vector of a moving body, enabling it to form a Hansen Ideal system with the basis of its coordinate system, is only its direction and not its value. Only the coordinates of its direction vector, and not the physical parameters of a vector, may be called Hansen Ideal coordinates with respect to the coordinate basis.

Theorem 9: The property of a vector to form a Hansen Ideal system with the basis of its coordinate system is restricted to the position vector, and therefore to the movement of the associated position. If a Hansen Ideal system can be constructed, this is only possible in connection with the motion of the related body.

\section{Necessity of Hansen Ideal coordinate systems}

In the following section, in extending the ideas of the original paper from Hansen, we would like to add some general but fundamental (and new) behaviour of Hansen Ideal coordinate systems. Based on Eq. (1) the value of the variation of the radial direction vector shall be defined by $\dot{\zeta}$. Then

$$
\boldsymbol{r}=r \boldsymbol{r}_{0}, \dot{\boldsymbol{r}}=\dot{r} \boldsymbol{r}_{0}+r \dot{\boldsymbol{r}}_{0}=\dot{r} \boldsymbol{r}_{0}+r \dot{\zeta} \boldsymbol{q}_{0}, \dot{\zeta}:=\left|\dot{\boldsymbol{r}}_{0}\right|
$$

Using these relations we derive the fundamental

Theorem 10: The Hansen Ideal coordinate system gets fixed unambiguously by assuming that the variation of the radial direction vector of a moving body is the derivative of an angle:

$$
\begin{aligned}
\dot{\boldsymbol{r}}_{0}= & \dot{\zeta} \boldsymbol{q}_{0}, \quad \dot{\zeta}=\left|\dot{\boldsymbol{r}}_{0}\right|, \quad\left(\boldsymbol{q}_{0}^{2}=1\right) \\
& \Leftrightarrow \quad \boldsymbol{q}_{j}^{(I)} \text {-system is Hansen Ideal. }
\end{aligned}
$$

Proof: 1. If

$\zeta=\zeta_{\mathrm{A}}+\int \dot{\zeta} \mathrm{d} t$

is understood as the angle coordinate within an orbital plane related $\boldsymbol{q}_{j}$-coordinate system and $\zeta_{\mathrm{A}}$ should be an initial value, then the radial and the transversal direction vector can be written as

$$
\begin{aligned}
& \boldsymbol{r}_{0}=\boldsymbol{q}_{1} \cos \zeta+\boldsymbol{q}_{2} \sin \zeta, \\
& \boldsymbol{q}_{0}=-\boldsymbol{q}_{1} \sin \zeta+\boldsymbol{q}_{2} \cos \zeta
\end{aligned}
$$

and vice versa

$$
\begin{aligned}
& \boldsymbol{q}_{1}=\boldsymbol{r}_{0} \cos \zeta-\boldsymbol{q}_{0} \sin \zeta, \\
& \boldsymbol{q}_{2}=\boldsymbol{r}_{0} \sin \zeta+\boldsymbol{q}_{0} \cos \zeta
\end{aligned}
$$

The variation will be

$$
\begin{aligned}
& \dot{\boldsymbol{q}}_{1}=\dot{\boldsymbol{r}}_{0} \cos \zeta-\dot{\boldsymbol{q}}_{0} \sin \zeta-\dot{\zeta}\left(\boldsymbol{r}_{0} \sin \zeta+\boldsymbol{q}_{0} \cos \zeta\right) \\
& \dot{\boldsymbol{q}}_{2}=\dot{\boldsymbol{r}}_{0} \sin \zeta+\dot{\boldsymbol{q}}_{0} \cos \zeta+\dot{\zeta}\left(\boldsymbol{r}_{0} \cos \zeta-\boldsymbol{q}_{0} \sin \zeta\right)
\end{aligned}
$$

Using the coordinates

$y_{1}=r \cos \zeta, \quad y_{2}=r \sin \zeta, \quad y_{3}=0$,

and with definition (21) we have

$\frac{1}{r} y^{j} \dot{\boldsymbol{q}}_{j}=\dot{\boldsymbol{r}}_{0}-\boldsymbol{q}_{0} \dot{\zeta}=0$,

i.e. the basic condition (9) of a Hansen system is fulfilled. Consequently, a coordinate system which is defined by the condition $\dot{\zeta}=\left|\dot{\boldsymbol{r}}_{0}\right|$ will necessarily be a Hansen Ideal coordinate system. Therefore in this case $\boldsymbol{q}_{j}=\boldsymbol{q}_{j}^{(I)}$.

2 . Vice versa for a given Hansen Ideal $\boldsymbol{q}_{j}^{(I)}$-coordinate system, based on the coordinate definition (27) it follows

$y^{j} \dot{\boldsymbol{q}}_{j}^{(I)}=r\left(\dot{\boldsymbol{q}}_{1}^{(I)} \cos \zeta+\dot{\boldsymbol{q}}_{2}^{(I)} \sin \zeta\right)=0$

and necessarily

$\dot{\boldsymbol{q}}_{1}^{(I)} \cos \zeta+\dot{\boldsymbol{q}}_{2}^{(I)} \sin \zeta=0$.

Therefore, from definition (24)

$$
\begin{aligned}
\dot{\boldsymbol{r}}_{0} & =\dot{\zeta}\left(-\boldsymbol{q}_{1}^{(I)} \sin \zeta+\boldsymbol{q}_{2}^{(I)} \cos \zeta\right)+ \\
& +\dot{\boldsymbol{q}}_{1}^{(I)} \cos \zeta+\dot{\boldsymbol{q}}_{2}^{(I)} \sin \zeta=\dot{\zeta} \boldsymbol{q}_{0},
\end{aligned}
$$

i.e. the condition (22) is fulfilled (q.e.d.).

An additional consequence from the previous theorem can we have from relations (21):

$$
\begin{aligned}
\boldsymbol{r} & \times \dot{\boldsymbol{r}}=\boldsymbol{c}=G \boldsymbol{c}_{0} r^{2} \boldsymbol{r}_{0} \times \dot{\boldsymbol{r}}_{0}= \\
& =r^{2} \dot{\zeta} \boldsymbol{r}_{0} \times \boldsymbol{q}_{0}=r^{2} \dot{\zeta} \boldsymbol{c}_{0} .
\end{aligned}
$$

The relation $r^{2} \dot{\zeta}=G$ is the well known scalar form of the equal area law. Based on Theorem 10 we get ${ }^{2}$

Theorem 11: The polar angle in the equal area law is only and only related to a Hansen system.

Usually, for each motion a radial and a transversal direction may be found. Therefore, another important consequence of Theorem 10 will be

Theorem 12: Hansen Ideal coordinate systems can be constructed for any motion.

Applying this result to orbital mechanics, a further theorem illuminates the central importance of the ideal coordinates systems detected by P. Hansen for the complete domain of astrodynamics. As a refinement of Theorem 11 with respect to reference systems in astrometry we get

Theorem 13: On the assumption that the basic reference system is inertial, the variation of the orbit angle occurring in the scalar form of Kepler's second law will necessarily lead to a Hansen Ideal coordinate system.

\footnotetext{
2 This issue might be seen to be contained in Palacios \& Calvo (1996, p. 69 ) however only in an implicit manner in the context of an improvement of numerical computation and not in the totally generalised and fundamental version as derived in the present paper.
} 


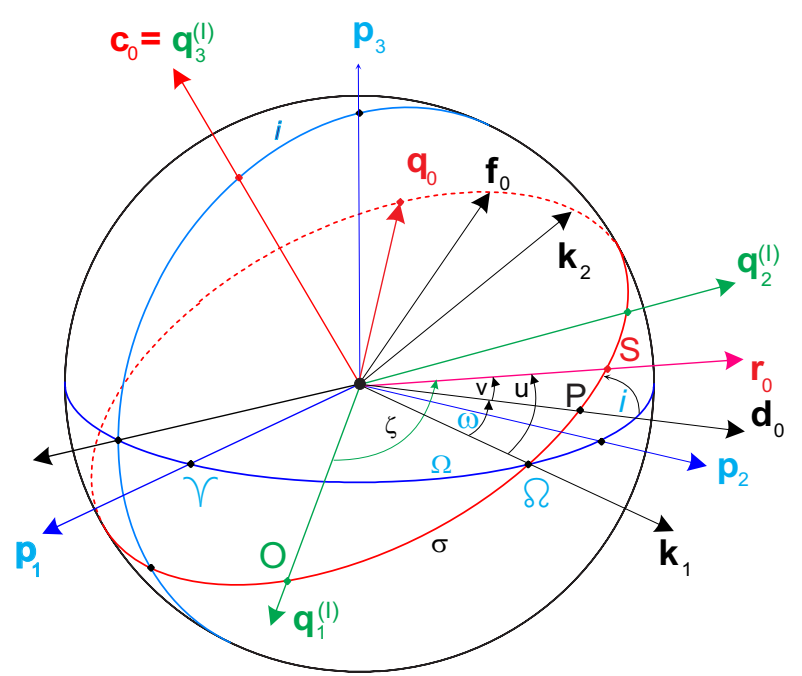

Fig. 1 (online colour at: www.an-journal.org) On the definition of the Hansen Ideal coordinate system. $\boldsymbol{p}_{1}, \boldsymbol{p}_{2}, \boldsymbol{p}_{3}$ - inertial fundamental system, $\boldsymbol{q}_{1}^{(\mathrm{I})}, \boldsymbol{q}_{2}^{(\mathrm{I})}, \boldsymbol{q}_{3}^{(\mathrm{I})}-$ Hansen system, $\boldsymbol{k}_{1}, \boldsymbol{k}_{2}, \boldsymbol{c}_{0}-$ node related coordinate system, $\boldsymbol{d}_{0}, \boldsymbol{f}_{0}, \boldsymbol{c}_{0}$ - apsides-related coordinate system, $\boldsymbol{r}_{0}, \boldsymbol{q}_{0}, \boldsymbol{c}_{0}$ - co-moving system (Leibniz-system), $\mathrm{V}$ - vernal equinox, $\mathrm{O}$ - departure point of the Hansen system, $\Omega$ - ascending node, $\mathrm{P}$ - pericentre (Perihelion, perigee, pericentre), $\Omega$ - right ascension (or longitude) of ascending node, $i$ - orbital inclination, $\sigma$ - longitude in orbit of ascending node, $\zeta$ - orbit angle, $\omega$ - argument of pericentre, $v$ - true anomaly, $u$ - argument of latitude.

Proof: According to Fig. 1 the orbital plane defined by the $\boldsymbol{q}_{j}$-system (which is not necessarily assumed to be a Hansen) shall be related to the $\boldsymbol{p}_{i}$-fundamental system which is assumed to be inertial: $\dot{\boldsymbol{p}}_{i} \equiv 0 . \Omega$ is the right ascension of the ascending node (or the longitude in an ecliptic system), rotation around the node vector $\boldsymbol{k}_{1}$ with inclination $i$ leads to the orbital plane. Here the celestial body has the position direction vector $\boldsymbol{r}_{0}$.

The orbit normal vector is given by $\boldsymbol{c}_{0}=\boldsymbol{r}_{0} \times \boldsymbol{q}_{0}=$ $\boldsymbol{q}_{1} \times \boldsymbol{q}_{2}$. According to Fig. 1 , with respect to the $\boldsymbol{p}_{i}$-system and using the argument of latitude $u$ as orbit angle, we get the well known defining vectors of the co-moving Leibnizsystem (in contrast to any other co-moving system we call this system after G. W. Leibniz (Aiton 1960) in the radial, transversal and normal direction:

$$
\begin{aligned}
\boldsymbol{r}_{0} & =\boldsymbol{p}_{1}(-\cos i \sin u \sin \Omega+\cos u \cos \Omega)+ \\
& +\boldsymbol{p}_{2}(\cos i \sin u \cos \Omega+\cos u \sin \Omega)+ \\
& +\boldsymbol{p}_{3} \sin i \sin u, \\
\boldsymbol{q}_{0} & =\boldsymbol{p}_{1}(-\cos i \cos u \sin \Omega-\sin u \cos \Omega)+ \\
& +\boldsymbol{p}_{2}(\cos i \cos u \cos \Omega-\sin u \sin \Omega)+ \\
& +\boldsymbol{p}_{3} \sin i \cos u, \\
\boldsymbol{c}_{0} & =\boldsymbol{p}_{1} \sin i \sin \Omega- \\
& -\boldsymbol{p}_{2} \sin i \cos \Omega+ \\
& +\boldsymbol{p}_{3} \cos i .
\end{aligned}
$$

The variation of the radial direction vector will be

$$
\begin{aligned}
\dot{\boldsymbol{r}}_{0} & =(\dot{u}+\dot{\Omega} \cos i) \boldsymbol{q}_{0}+ \\
& +[(i) \cdot \sin u-\dot{\Omega} \cos u \sin i] \boldsymbol{c}_{0}, \\
\dot{\boldsymbol{p}}_{i} & \equiv 0 .
\end{aligned}
$$

The area law gives

$$
\begin{aligned}
\boldsymbol{r} & \times \dot{\boldsymbol{r}}=\boldsymbol{c}=G \boldsymbol{c}_{0}=r^{2}(\dot{u}+\dot{\Omega} \cos i) \boldsymbol{c}_{0}+ \\
& +r^{2}\left[(i)^{\cdot} \sin u-\dot{\Omega} \cos u \sin i\right] \boldsymbol{r}_{0} \times \boldsymbol{c}_{0},
\end{aligned}
$$

so that

$(i)^{\cdot} \sin u=\dot{\Omega} \cos u \sin i, \quad \dot{\boldsymbol{p}}_{i} \equiv 0$, and finally

$G=r^{2}(\dot{u}+\dot{\Omega} \cos i), \quad \dot{\boldsymbol{p}}_{i} \equiv 0$.

Using, as usual in orbital mechanics, the argument of latitude in the form $u=: v+\omega$, where $v$ is the true anomaly and $\omega$ the argument of the pericentre, we have

$G=r^{2}(\dot{v}+\dot{\omega}+\dot{\Omega} \cos i)$.

Defining

$\dot{\zeta}:=\dot{u}+\dot{\Omega} \cos i, \quad \dot{\boldsymbol{p}}_{i} \equiv 0$,

the condition $\dot{\boldsymbol{r}}_{0}=\dot{\zeta} \boldsymbol{q}_{0}$ is always fulfilled. Therefore, Theorem 10 can be applied in this general case of the motion of a celestial body. The orbit angle defined by Eq. (39) leads necessarily to a Hansen Ideal coordinate system, assuming that the basic reference system, to which the orbit parameters $i, \Omega, \omega$ are related, is inertial (q.e.d.).

Based on this theorem, it could be assumed that Hansen came upon the idea of an "ideal" frame as the result of his investigations on perturbation theory. However, no hint of this is to be found in his original paper. Therefore, it can be assumed that Hansen extended his discovery to any parameter, so that his original discovery was effaced, especially by using the obscure, non-vector style.

Hansen himself calls the orbit angle $\zeta$ together with the radius $r$, "ideal" parameters. However, this issue is of no mathematical relevance except perhaps meaning that the orbit angle $\zeta$ might be used as an independent variable instead of time (as consequence, we could also call this angle the "first Hansen angle").

The ascending node has an angular distance from the departure point $\mathrm{O}$, the longitude in orbit $\sigma$, which will be introduced by the differential equation

$\dot{\sigma}:=\dot{\Omega} \cos i$,

so that the orbit angle can be represented by

$\zeta=v+\omega+\sigma$.

In this context, there are two special cases to be added:

1. If the argument of pericentre $\omega$ and the right ascension of the ascending node $\Omega$ do not show any variation ( $\dot{\omega}=\dot{\Omega} \equiv 0$ ), the orbit angle will be reduced to the true anomaly. In this case the motion related orbital system is reduced to the $\boldsymbol{d}_{0}, \boldsymbol{f}_{0},\left(\boldsymbol{c}_{0}\right)$-apsidal system. In this context (and only in this context) the apsidal system might be assumed to be an "ideal" system. This means that the general property of the Hansen Ideal coordinate system includes the "unperturbed" case if no accelerations influence the orientation of the orbital plane in space. 
2. In a similar manner, the nodal system might be called a Hansen Ideal coordinate system if, and only if, the position of the node is inertially fixed $(\dot{\Omega} \equiv 0)$.

In most investigations a proper motion of the $\boldsymbol{p}_{i}$-basic system will not be included. However, in the context of Hansen Ideal coordinate systems an interesting feature could be detected. Based on the representation of the radial direction vector in fundamental coordinates in Eq. (33) the variation will be

$$
\begin{aligned}
\dot{\boldsymbol{r}}_{0} & =\dot{\boldsymbol{p}}_{1}(-\cos i \sin u \sin \Omega+\cos u \cos \Omega)+ \\
& +\dot{\boldsymbol{p}}_{2}(\cos i \sin u \cos \Omega+\cos u \sin \Omega)+ \\
& +\dot{\boldsymbol{p}}_{3} \sin i \sin u+ \\
& +\boldsymbol{p}_{1}[(i) \cdot \sin i \sin u \sin \Omega+ \\
& +\dot{u}(-\cos i \cos u \sin \Omega-\sin u \cos \Omega)+ \\
& +\dot{\Omega}(-\cos i \sin u \cos \Omega-\cos u \sin \Omega)]+ \\
& +\boldsymbol{p}_{2}[-(i) \cdot \sin i \sin u \cos \Omega+ \\
& +\dot{u}(\cos i \cos u \cos \Omega-\sin u \sin \Omega)+ \\
& +\dot{\Omega}(-\cos i \sin u \sin \Omega+\cos u \cos \Omega)]+ \\
& +\boldsymbol{p}_{3}[(i) \cdot \cos i \sin u+\dot{u} \sin i \cos u] .
\end{aligned}
$$

With the proper motion of the $\boldsymbol{p}_{i}$-system given by Eq. (4) and introducing the definition

$\dot{\zeta}_{\mathrm{B}}:=\dot{v}+\dot{\omega}+\dot{\Omega} \cos i$,

(the index $\mathrm{B}$ refers to the non-inertial basic system), the variation of the radial direction vector will be

$$
\begin{aligned}
& \dot{\boldsymbol{r}}_{0}=\dot{\zeta}_{\mathrm{B}} \boldsymbol{q}_{0}+\boldsymbol{c}_{0}[(i) \cdot \sin u-\dot{\Omega} \cos u \sin \Omega]+ \\
& \quad+\boldsymbol{q}_{0}\left(p_{12} \cos i+p_{23} \sin i \sin \Omega+\right. \\
& \left.\quad+p_{13} \sin i \cos \Omega\right)+ \\
& \quad+\boldsymbol{c}_{0}\left[-p_{12} \sin i \cos u+\right. \\
& \quad+p_{13}(\cos i \cos u \cos \Omega-\sin u \sin \Omega)+ \\
& \left.\quad+p_{23}(\cos i \cos u \sin \Omega+\sin u \cos \Omega)\right] .
\end{aligned}
$$

Using the absolute proper motion vector of the (general, i.e. not necessarily inertial) $\boldsymbol{p}_{i}$-system

$\boldsymbol{D}_{p}=D_{p}^{i} \boldsymbol{p}_{i}=p_{23} \boldsymbol{p}_{1}+p_{31} \boldsymbol{p}_{2}+p_{12} \boldsymbol{p}_{3}$,

the variation of the radial direction vector becomes

$$
\begin{aligned}
\dot{\boldsymbol{r}}_{0} & =\boldsymbol{q}_{0}\left(\dot{\zeta}_{\mathrm{B}}+\boldsymbol{D}_{p} \cdot \boldsymbol{c}_{0}\right)+ \\
& +\boldsymbol{c}_{0}\left\{[(i) \cdot \sin u-\dot{\Omega} \cos u \sin i]-\boldsymbol{D}_{p} \cdot \boldsymbol{q}_{0}\right\} .
\end{aligned}
$$

Because $\dot{\boldsymbol{r}}_{0} \cdot \boldsymbol{c}_{0}=0$, the condition

$\dot{\boldsymbol{r}}_{0}=\boldsymbol{q}_{0}\left(\dot{\zeta}_{\mathrm{B}}+\boldsymbol{D}_{p} \cdot \boldsymbol{c}_{0}\right)=\dot{\zeta} \boldsymbol{q}_{0}$

remains with the additional condition

(i) $\sin u-\dot{\Omega} \cos u \sin i=\boldsymbol{D}_{p} \cdot \boldsymbol{q}_{0}$.

In this more general case, the variation of the orbit angle will be presented by

$\dot{\zeta}=\left|\dot{\boldsymbol{r}}_{0}\right|=\dot{\zeta}_{\mathrm{B}}+\boldsymbol{D}_{p} \cdot \boldsymbol{c}_{0}=: \dot{\zeta}_{\mathrm{B}}+\dot{\zeta}_{\mathrm{P}}$

in relation to a Hansen Ideal coordinate system, however, now including the proper motion of the basic reference system. The result will be summarized in

Theorem 14: If the motion of a celestial body is related to a basic system which is not an inertial system, the proper motion of this system will be reflected in the orbit angle by its relation to its Hansen Ideal coordinate system.

The consequence of this theorem could be remarkable: if it were possible to measure independently the variation of the orbit angle (e.g. of an artificial Earth satellite) from the variation of the radial direction vector $\left(\dot{\zeta}=\left|\dot{\boldsymbol{r}}_{0}\right|\right)$ and the variation of the angle related to the basic system (from $\dot{\zeta}_{\mathrm{B}}:=\dot{v}+\dot{\omega}+\dot{\Omega} \cos i$ ), then as a consequence it might be expected to be able to measure the absolute system proper motion vector $\boldsymbol{D}_{p}$ of the basic system by means of the difference of the variation of the orbit angles $\dot{\zeta}_{\mathrm{P}}=\dot{\zeta}-\dot{\zeta}_{\mathrm{B}}=$ $\boldsymbol{D}_{p} \cdot \boldsymbol{c}_{0}$. In this way, a confirmation of an inertial fundamental system would be possible. In order to compute $\dot{\zeta}=\left|\dot{\boldsymbol{r}}_{0}\right|$ from Eq. (35) the scalar form of the equal area relation will be

$\dot{\zeta}=\frac{G}{r^{2}}$

which is independent of the proper motion of the $\boldsymbol{p}_{i}$-basic reference system ("inertial fundamental system"). In order to calculate $\dot{\zeta}_{\mathrm{B}}:=\dot{v}+\dot{\omega}+\dot{\Omega} \cos i$, the instantaneous values for $\dot{u}=\dot{v}+\dot{\omega}$ and $\dot{\Omega}$ may be computed using the usual Gaussian or Lagrangean variational equations of orbital mechanics.

Note: The previous considerations are restricted to basic mathematical insights only. However, there is not at all any statement in principle concerning the physical or even technical realisation of such an independent measurement of the variation of the transversal vector of motion.

\section{Proper motion of the Leibniz system}

The orbital system which is moving with the celestial body is defined by the radial, the transversal, and the normal direction vector. (This system is sometimes referred to as an HCL-system, where $\mathrm{L}=$ longtrack is perpendicular to the radial direction and not coinciding with the velocity vector). The acceleration which is responsible for the motion of a celestial body is represented in the co-moving orbital system by

$\ddot{\boldsymbol{r}}=b_{\mathrm{R}} \boldsymbol{r}_{0}+b_{\mathrm{T}} \boldsymbol{q}_{0}+b_{\mathrm{N}} \boldsymbol{c}_{0}$,

where $b_{\mathrm{R}}$ is the radial, $b_{\mathrm{T}}$ the transversal, and $b_{\mathrm{N}}$ the normal part of the acceleration. With respect to the Hansen Ideal $\boldsymbol{q}_{j}$-coordinate system, Eq. (21) leads to

$\ddot{\boldsymbol{r}}=\ddot{r} \boldsymbol{r}_{0}+2 \dot{r} \dot{\zeta} \boldsymbol{q}_{0}+r \ddot{\zeta} \boldsymbol{q}_{0}+r \dot{\zeta} \dot{\boldsymbol{q}}_{0}$.

Because $\boldsymbol{q}_{0} \cdot \dot{\boldsymbol{q}}_{0}=0$, the assessment

$\dot{\boldsymbol{q}}_{0}=\dot{\xi} \boldsymbol{r}_{0}+\dot{\eta} \boldsymbol{c}_{0}$

with certain parameters $\dot{\xi}, \dot{\eta}$ is allowed. Then

$\ddot{\boldsymbol{r}}=(\ddot{r}+r \dot{\zeta} \dot{\xi}) \boldsymbol{r}_{0}+(2 \dot{r} \dot{\zeta}+r \ddot{\zeta}) \boldsymbol{q}_{0}+r \dot{\zeta} \dot{\eta} \boldsymbol{c}_{0}$.

The transversal direction vector in Eq. (24) has the variation $\begin{aligned} \dot{\boldsymbol{q}}_{0}= & -\dot{\zeta}\left(\boldsymbol{q}_{1}^{(\mathrm{I})} \cos \zeta+\boldsymbol{q}_{2}^{(\mathrm{I})} \sin \zeta\right)- \\ & -\dot{\boldsymbol{q}}_{1}^{(\mathrm{I})} \sin \zeta+\dot{\boldsymbol{q}}_{2}^{(\mathrm{I})} \cos \zeta\end{aligned}$ 
Because of the Hansen Ideal behaviour of the $\boldsymbol{q}_{j}^{(\mathrm{I})}$-system with Eq. (30), the system variational vectors $\dot{\boldsymbol{q}}_{1}^{(\mathrm{I})}$ and $\dot{\boldsymbol{q}}_{2}^{(\mathrm{I})}$ are collinear with the orbit normal vector $\boldsymbol{c}_{0}$. Using $\boldsymbol{r}_{0}$ from Eq. (24), it follows

$\dot{\boldsymbol{q}}_{0}=-\dot{\zeta} \boldsymbol{r}_{0}-\left(\left|\dot{\boldsymbol{q}}_{1}^{(\mathrm{I})}\right| \sin \zeta-\left|\dot{\boldsymbol{q}}_{2}^{(\mathrm{I})}\right| \cos \zeta\right) \boldsymbol{c}_{0}$.

Comparison with Eq. (53) gives

$\dot{\xi}=-\dot{\zeta}, \quad \dot{\eta} \boldsymbol{c}_{0}=-\dot{\boldsymbol{q}}_{1}^{(I)} \sin \zeta+\dot{\boldsymbol{q}}_{2}^{(I)} \cos \zeta$.

With this expression, a comparison of Eqs. (51) and (56) leads to the condition of the radial acceleration,

$b_{\mathrm{R}}=\ddot{r}-r \dot{\zeta}^{2}=\ddot{r}-\frac{G^{2}}{r^{3}}$,

which is a relation known as (general) Leibniz equation (cf. Aiton 1960). Leibniz used the Keplerian acceleration $b_{\mathrm{R}}=$ $-G^{2} /\left(p r^{2}\right)$. The transversal acceleration reads

$b_{\mathrm{T}}=2 \dot{r} \dot{\zeta}+r \ddot{\zeta}$.

By using $G=r^{2} \dot{\zeta}$ from Eq. (50), it follows

$\dot{G}=r b_{\mathrm{T}}$.

Because $\zeta$ is a geometrical parameter, it might be assumed that also $\eta$ would be a geometrical parameter. This question will be answered in the next section. In any case, $\eta$ seems to be a parameter in intimate contact with the Hansen Ideal coordinate system. From Eqs. (51) and (56) we denote the influence of the normal acceleration by

$b_{\mathrm{N}}=r \dot{\zeta} \dot{\eta}=\frac{G}{r} \dot{\eta}$.

Because $\boldsymbol{r}_{0}, \boldsymbol{q}_{0}, \boldsymbol{c}_{0}$ is the orthonormal basis of an associated, moving, orbital system, the variational equation for the variation $\dot{\boldsymbol{c}}_{0}$ of the normal direction vector can be completed according to the variational Eqs. (3). Therefore,

Theorem 15: With respect to a Hansen Ideal, orbit related coordinate system, the Leibniz system (as a co-moving orbital system) has the Frenet equations

$$
\begin{aligned}
& \dot{\boldsymbol{r}}_{0}=\dot{\zeta} \boldsymbol{q}_{0}, \\
& \dot{\boldsymbol{q}}_{0}=-\dot{\zeta} \boldsymbol{r}_{0}+\dot{\eta} \boldsymbol{c}_{0}, \\
& \dot{\boldsymbol{c}}_{0}=-\dot{\eta} \boldsymbol{q}_{0} \text {. }
\end{aligned}
$$

Thus we find, in analogy to curvature theory, a kind of Frenet trihedron in orbital mechanics, describing the motion of a celestial body. This orbital system related trihedron is intimately connected with the Hansen Ideal coordinate system. It will not exist without relation to a Hansen system. (This trihedron could be called the "astrodynamics accompanying trihedron"). The absolute system proper motion vector (Darboux vector) of the Leibniz system can be derived in a corresponding manner:

Theorem 16: With respect to a Hansen Ideal orbit related coordinate system, a Leibniz system has the proper motion vector

$\boldsymbol{D}_{q^{(\mathrm{L})}}:=\dot{\eta} \boldsymbol{r}_{0}+\dot{\zeta} \boldsymbol{c}_{0}$.

Consequently, the following variational equations read

$$
\begin{aligned}
& \dot{\boldsymbol{r}}_{0}=\boldsymbol{D}_{q^{(\mathrm{L})}} \times \boldsymbol{r}_{0}, \\
& \dot{\boldsymbol{q}}_{0}=\boldsymbol{D}_{q^{(\mathrm{L})}} \times \boldsymbol{q}_{0}, \\
& \dot{\boldsymbol{c}}_{0}=\boldsymbol{D}_{q^{(\mathrm{L})}} \times \boldsymbol{c}_{0} .
\end{aligned}
$$

Because $\dot{\boldsymbol{r}}=\dot{r} \boldsymbol{r}_{0}+r \dot{\boldsymbol{r}}_{0}$, where in general $r \dot{\boldsymbol{r}}_{0} \neq 0$, the conclusion is

Theorem 17: The $\boldsymbol{r}_{0}, \boldsymbol{q}_{0}, \boldsymbol{c}_{0}$-Leibniz system is not a Hansen Ideal system.

Note: Theorems 15 and 16 show the (surprisingly) close relationship between the Leibniz and Hansen systems.

\section{Proper motion of the Hansen Ideal coordinate system}

The second of Eqs. (57) together with condition (30) results in

$\dot{\boldsymbol{q}}_{1}^{(\mathrm{I})}=-\boldsymbol{c}_{0} \dot{\eta} \sin \zeta, \quad \dot{\boldsymbol{q}}_{2}^{(\mathrm{I})}=\boldsymbol{c}_{0} \dot{\eta} \cos \zeta$.

Using the variational Eqs. (3), the previous system will be completed by the corresponding variational equation for the third direction vector $\boldsymbol{q}_{3}=\boldsymbol{c}_{0}=\boldsymbol{q}_{1} \times \boldsymbol{q}_{2}$. Then

Theorem 18: The Hansen Ideal orbit related coordinate system has the variational equations

$\begin{array}{lr}\dot{\boldsymbol{q}}_{1}^{(I)}= & -\dot{\eta} \boldsymbol{q}_{3}^{(\mathrm{I})} \sin \zeta, \\ \dot{\boldsymbol{q}}_{2}^{(\mathrm{I})}= & \dot{\eta} \boldsymbol{q}_{3}^{(\mathrm{I})} \cos \zeta, \\ \dot{\boldsymbol{q}}_{3}^{(I)}=\dot{\eta}\left(\boldsymbol{q}_{1}^{(\mathrm{I})} \sin \zeta-\boldsymbol{q}_{2}^{(\mathrm{I})} \cos \zeta\right) .\end{array}$

Similarly,

Theorem 19 (extension of Hansen 1857): A coordinate system is a Hansen Ideal system, if, and only if, its system proper motion vector has the form

$\boldsymbol{D}_{q^{(\mathrm{I})}}=\dot{\eta} \boldsymbol{r}_{0}$.

Proof: Eq. (67) results from Eq. (66), so

$\dot{\boldsymbol{q}}_{j}^{(\mathrm{I})}=\boldsymbol{D}_{q^{(\mathrm{I})}} \times \boldsymbol{q}_{j}^{(\mathrm{I})}$.

Vice versa, if $\boldsymbol{D}_{q^{(\mathrm{I})}}=\dot{\eta} \boldsymbol{r}_{0}$, Eq. (62) follow via condition (68). Then, with $y_{1}=r \cos \zeta, y_{2}=r \sin \zeta, y_{3}=0$,

$$
\begin{aligned}
& y^{j} \dot{\boldsymbol{q}}_{j}^{(\mathrm{I})}=r \cos \zeta\left(-\dot{\eta} \boldsymbol{q}_{3}^{(\mathrm{I})} \sin \zeta\right)+ \\
& +r \sin \zeta\left(\dot{\eta} \boldsymbol{q}_{3}^{(\mathrm{I})} \cos \zeta\right)=0 .
\end{aligned}
$$

The condition of Theorem 1 is fulfilled (q.e.d.).

Equation (67) specifies relation (20) in detail. Obviously $\dot{\eta}=A r$, so that

$$
\begin{gathered}
q_{12}=D_{q 3}=\frac{\dot{\eta}}{r} y_{3} \\
q_{23}=D_{q 1}=\frac{\dot{\eta}}{r} y_{1} \\
q_{31}=D_{q 2}=\frac{\dot{\eta}}{r} y_{2} .
\end{gathered}
$$

The last two theorems lead to

Theorem 20: A Hansen Ideal system has a proper motion only if a normal acceleration influences the motion of the related body. Otherwise it will be inertially fixed.

Conclusions: (1) If no variations in a Hansen Ideal system can be observed, i.e. if there is no variation $\dot{\eta}$ of the hidden rotational angle $\eta$, this system will be inertially fixed.

(2) The acceleration causing a Hansen Ideal system to be non-inertial is only perpendicularly influencing the orbital 


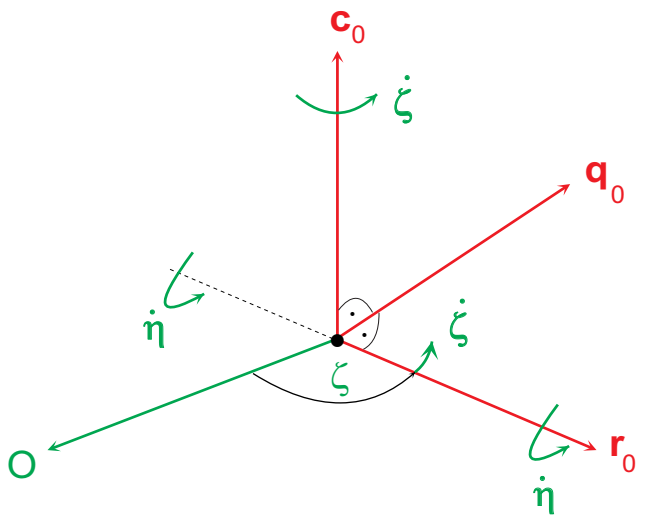

Fig. 2 (online colour at: www.an-journal.org) Geometrical interpretation of the second angle coordinate of a Hansen Ideal coordinate system.

plane of a moving celestial body (or an artificial Earth satellite).

(3) In the case of a Hansen system, usually the third Cartesian coordinate is assumed to vanish: $y_{3} \equiv 0$. Then it follows from Eq. (70) $q_{12}=D_{q 3}=0$, and we have ${ }^{3}$

Theorem 21: The third component of the absolute proper motion vector of a Hansen system vanishes identically.

This is a necessary and sufficient condition, as easily will be seen by an indirect proof.

Now Hansen's Theorem 5 in Eq. (16) can be made more precise: A Hansen Ideal coordinate system rotates only around the radius vector $\boldsymbol{r}$. Equation (67) shows this rotation has the angular velocity $\dot{\eta}$. This leads to the geometrical interpretation of this parameter as a hidden angle, which can be observed by its variation only (cf. Fig. 2). By means of the variational Eqs. (62), this parameter is closely related to the Hansen Ideal system and therefore could be called the second angle coordinate of a Hansen Ideal coordinate system. However, this second angle is not an independent parameter but a function of the orbit angle $\zeta$.

In order to derive a relation between $\dot{\eta}$ and the variation of the orbit orientation angles i, $\Omega, \sigma$, (see Fig. 1), the direction vectors of the node oriented orbital plane system will be used:

$$
\begin{aligned}
\boldsymbol{k}_{1} & =\boldsymbol{p}_{1} \cos \Omega+\boldsymbol{p}_{2} \sin \Omega=\boldsymbol{q}_{1} \cos \sigma+\boldsymbol{q}_{2} \sin \sigma, \\
\boldsymbol{k}_{2} & =-\boldsymbol{p}_{1} \cos i \sin \Omega+\boldsymbol{p}_{2} \cos i \cos \Omega+\boldsymbol{p}_{3} \sin i \\
& =-\boldsymbol{q}_{1} \sin \sigma+\boldsymbol{q}_{2} \cos \sigma .
\end{aligned}
$$

The orbit normal vector $\mathbf{c}$ from Eq. (33) and the third of the variational equations in Eqs. (62) give

$$
\begin{aligned}
\dot{\boldsymbol{c}}_{0}= & -\dot{\eta} \boldsymbol{q}_{0}=\dot{\boldsymbol{p}}_{1} \sin i \sin \Omega- \\
& -\dot{\boldsymbol{p}}_{2} \sin i \cos \Omega+\dot{\boldsymbol{p}}_{3} \cos i+ \\
& +(i) \cdot\left[\boldsymbol{p}_{1} \cos i \sin \Omega-\right. \\
& \left.-\boldsymbol{p}_{2} \cos i \cos \Omega-\boldsymbol{p}_{3} \sin i\right]+ \\
& +\dot{\Omega}\left[\boldsymbol{p}_{1} \cos \Omega+\boldsymbol{p}_{2} \sin \Omega\right] .
\end{aligned}
$$

The transversal direction vector with respect to the $\boldsymbol{p}_{i}$ fundamental system (33), the variational Eqs. (3) of the ba-

\footnotetext{
3 See also Deprit (1976).
}

sic system, the general Darboux vector $D_{p}$ in Eq. (4) together with the relationships (6), yield

$$
\begin{aligned}
(i) & =\dot{\eta} \cos u-\boldsymbol{D}_{P} \cdot \boldsymbol{k}_{1}, \\
\dot{\Omega} \sin i & =\dot{\eta} \sin u-\boldsymbol{D}_{P} \cdot \boldsymbol{k}_{2},
\end{aligned}
$$

and

(i) $\cdot \sin u-\dot{\Omega} \cos u \sin i=\boldsymbol{D}_{p} \cdot \boldsymbol{q}_{0}$.

The angle $\eta$ is found e.g. in Stumpff (1974, p. 200) as the angle between two instantaneous orbital planes. The differential relations between the rotational angle $\eta$ and the orientation angles $i$ and $\Omega$, including the interpretation of the angle $\eta$, are firstly found in known literature in Struble (1961, p. 89), however, without reference to the proper motion of the basic system. The variational Eqs. (73) and (74) will be completed by the condition for the longitude of the node in the orbital plane which is based on the variation of the nodal vector (71) in both systems; finally, using the normal vector (33), the relation

$\dot{\sigma}=\dot{\Omega} \cos i+\boldsymbol{D}_{p} \cdot \boldsymbol{c}_{0}$

will be obtained. The relative transformation of the velocity vector from the Hansen Ideal $\boldsymbol{q}_{j}^{(\mathrm{I})}$-system to a $\boldsymbol{p}_{i}$-basic system,

$\dot{\boldsymbol{r}}_{p}=\dot{\boldsymbol{r}}_{q}-\boldsymbol{D}_{p} \times \boldsymbol{r}$,

reflects the proper motion of this basic system, thus proving Theorem 14 of the Hansen theory.

Note: The basic $\boldsymbol{p}_{i}$-system may not necessarily be assumed to be inertial. ,

Remark: The vector $\dot{\boldsymbol{r}}_{q p}:=\dot{\boldsymbol{r}}_{q}-\dot{\boldsymbol{r}}_{p}=\boldsymbol{D}_{p} \times \boldsymbol{r}$ will be called the "relative system proper motion vector" of the $\boldsymbol{q}_{j}^{(\mathrm{I})}$-system with respect to the $\boldsymbol{p}_{i}$-system. In this context, the Euler angles $\Omega, i, \sigma$ represent the relative transformation of the $\boldsymbol{q}_{j}^{(\mathrm{I})}$-Hansen system into the basic $\boldsymbol{p}_{i}$-system.

\section{The Hansen system and the method of variation of parameters}

Let any orbital curve be defined by the polar equation

$r=r\left(A_{k}^{(\nu)} ; \zeta\right)$.

Here the parameters $A_{k}^{(\nu)}$ define the form of the curve under consideration and $\zeta$ is the (first Hansen) orbit angle. Then the radial velocity reads

$\dot{r}=\frac{\mathrm{d} r}{\mathrm{~d} \zeta} \dot{\zeta}=\left(\frac{\partial r}{\partial \zeta}+\sum_{k=1}^{K} \frac{\partial r}{\partial A_{k}^{(\nu)}} \frac{\mathrm{d} A_{k}^{(\nu)}}{\mathrm{d} \zeta}\right) \dot{\zeta}$.

The Hansen Ideal condition, that perturbed motion should be represented in the same form as unperturbed motion, leads to the requirement

$\frac{d r}{d \zeta}=\frac{\partial r}{\partial \zeta}$.

Therefore,

$\sum_{k=1}^{K} \frac{\partial r}{\partial A_{k}^{(\nu)}} \frac{\mathrm{d} A_{k}^{(\nu)}}{\mathrm{d} \zeta}=0$. 
This is the osculation condition (Lagrange constraint) ${ }^{4}$ as used in the method of the variation of the parameters. As a consequence, we have

Theorem 22: The Lagrange constraint fulfils necessarily the Hansen Ideal condition.

In order to extent this theorem, we would like to introduce an arbitrary orbit related rectangular coordinate system using the orthonormal basic vectors $\boldsymbol{q}_{j}^{(\mathrm{B})}$, whose first two basic vectors are correlated to the instantaneous orbital plane. $\psi$ should be the angular distance of the departure point of this system with respect to the radius vector. Therefore the relation to the Leibniz system holds:

$\boldsymbol{r}_{0}=\boldsymbol{q}_{1}^{(\mathrm{B})} \cos \psi+\boldsymbol{q}_{2}^{(\mathrm{B})} \sin \psi$,

$\boldsymbol{q}_{0}=-\boldsymbol{q}_{1}^{(\mathrm{B})} \sin \psi+\boldsymbol{q}_{2}^{(\mathrm{B})} \cos \psi$,

$c_{0}=\boldsymbol{q}_{3}^{(\mathrm{B})}=\boldsymbol{q}_{1}^{(\mathrm{B})} \times \boldsymbol{q}_{2}^{(\mathrm{B})}$,

$\boldsymbol{q}_{1}^{(\mathrm{B})}=\boldsymbol{r}_{0} \cos \psi-\boldsymbol{q}_{0} \sin \psi$,

$\boldsymbol{q}_{2}^{(\mathrm{B})}=\boldsymbol{r}_{0} \sin \psi+\boldsymbol{q}_{0} \cos \psi$,

$\boldsymbol{q}_{3}^{(\mathrm{B})}=\boldsymbol{c}_{0}=\boldsymbol{r}_{0} \times \boldsymbol{q}_{0}$.

By means of the Frenet formulae (24) we have

$$
\begin{aligned}
& \dot{\boldsymbol{q}}_{1}^{(\mathrm{B})}=\quad(\dot{\zeta}-\dot{\psi}) \dot{\boldsymbol{q}}_{2}^{(\mathrm{B})}+\dot{\eta} \mathbf{q}_{3}^{(\mathrm{B})} \sin \psi, \\
& \dot{\boldsymbol{q}}_{2}^{(\mathrm{B})}=-(\dot{\zeta}-\dot{\psi}) \mathbf{q}_{1}^{(\mathrm{B})}+\dot{\eta} \mathbf{q}_{3}^{(\mathrm{B})} \cos \psi, \\
& \dot{\boldsymbol{q}}_{3}^{(\mathrm{B})}=\dot{\eta}\left(\boldsymbol{q}_{1}^{(\mathrm{B})} \sin \psi-\boldsymbol{q}_{2}^{(\mathrm{B})} \cos \psi\right) .
\end{aligned}
$$

Such a system is not necessarily a Hansen system, so that in general $\psi \neq \zeta$ and $\dot{\psi} \neq \dot{\zeta}$ has to be taken into account. The system proper motion vector of this system,

$\boldsymbol{D}_{q^{(\mathrm{B})}}=: D_{q^{(\mathrm{B})}}^{j} \boldsymbol{q}_{j}^{(\mathrm{B})}$,

has evidently the components

$$
\begin{aligned}
& D_{q^{(\mathrm{B})} 1}=\dot{\eta} \cos \psi, \\
& D_{q^{(\mathrm{B})} 2}=\dot{\eta} \sin \psi, \\
& D_{q^{(\mathrm{B})} 3}=\dot{\zeta}-\dot{\psi} .
\end{aligned}
$$

Therefore, with the first of the relations (81) we have

$$
\begin{aligned}
\boldsymbol{D}_{q^{(\mathrm{B})}}= & \dot{\eta}\left(\boldsymbol{q}_{1}^{(\mathrm{B})} \cos \psi+\boldsymbol{q}_{2}^{(\mathrm{B})} \sin \psi\right)+ \\
& +(\dot{\zeta}-\dot{\psi}) \boldsymbol{q}_{3}^{(\mathrm{B})}=\dot{\eta} \boldsymbol{r}_{0}+(\dot{\zeta}-\dot{\psi}) \boldsymbol{c}_{0} .
\end{aligned}
$$

The latter relation forms another very simple proof to Theorem 19. A consequence will be

Theorem 23: The Hansen system is the only system related to the osculating plane of an orbital motion, which is absolutely fixed within this plane.

Proof: If the system is a Hansen system, then $\dot{\psi}=\dot{\zeta}$ and the relations (82) show no variation of the first two basic vectors within the orbital plane. Indirectly vice versa: Assuming the $\boldsymbol{q}_{j}^{(\mathrm{B})}$-system not being a Hansen system, then $\dot{\psi} \neq \dot{\zeta}$ and the $\boldsymbol{q}_{j}^{(\mathrm{B})}$-system has a proper motion relatively to the orbital plane with the angular velocity $\dot{\zeta}-\dot{\psi} \neq 0$. Consequently, such a system cannot be fixed with respect to the orbital plane (q.e.d.).

\footnotetext{
${ }^{4}$ See, e.g., in Efroimsky (2005).
}

Remark: In Theorem 7 we have seen that each motion of a moving body in infinite number of possible Hansen Ideal coordinate systems could be constructed. Based on Theorem 23 we may assess that besides for an arbitrary integrational constant $\zeta_{\mathrm{A}}$ (see the integral 23) there is in principle only one Hansen system connected to any motion.

As a consequence of Theorem 23 we would like to investigate its relation to the Lagrange constraint. The position vector of a moving object within a $\boldsymbol{q}_{j}^{(\mathrm{B})}$-system might be represented by

$\boldsymbol{r}=r \boldsymbol{r}_{0}=y^{j} \boldsymbol{q}_{j}^{(\mathrm{B})}=r\left(\boldsymbol{q}_{1}^{(\mathrm{B})} \cos \psi+\boldsymbol{q}_{2}^{(\mathrm{B})} \sin \psi\right)$.

Its absolute variation with respect to the orbital angle $\psi$ is

$$
\begin{aligned}
\frac{\mathrm{d} \boldsymbol{r}}{\mathrm{d} \psi}= & \frac{\mathrm{d} r}{\mathrm{~d} \psi} \boldsymbol{r}_{0}+ \\
& +r\left(\frac{\mathrm{d} \boldsymbol{q}_{1}^{(\mathrm{B})}}{\mathrm{d} \psi} \cos \psi+\frac{\mathrm{d} \boldsymbol{q}_{2}^{(\mathrm{B})}}{\mathrm{d} \psi} \sin \psi\right)+r \boldsymbol{q}_{0},
\end{aligned}
$$

its relative variation using partial differentiation

$$
\begin{aligned}
\frac{\partial \boldsymbol{r}}{\partial \psi}= & \frac{\partial r}{\partial \psi} \boldsymbol{r}_{0}+ \\
& +r\left(\frac{\partial \boldsymbol{q}_{1}^{(\mathrm{B})}}{\partial \psi} \cos \psi+\frac{\partial \boldsymbol{q}_{2}^{(\mathrm{B})}}{\partial \psi} \sin \psi\right)+r \boldsymbol{q}_{0} .
\end{aligned}
$$

The orbit angle $\psi$ is related to the $\boldsymbol{q}_{j}^{(\mathrm{B})}$-system. Therefore

$$
\frac{\partial \boldsymbol{q}_{1}^{(\mathrm{B})}}{\partial \psi}=\frac{\partial \boldsymbol{q}_{2}^{(\mathrm{B})}}{\partial \psi}=0 .
$$

The difference between the absolute and partial variation of the position vector is

$$
\begin{aligned}
\frac{\mathrm{d} \boldsymbol{r}}{\mathrm{d} \psi}-\frac{\partial \boldsymbol{r}}{\partial \psi} & =\left(\frac{\mathrm{d} r}{\mathrm{~d} \psi}-\frac{\partial r}{\partial \psi}\right) \boldsymbol{r}_{0}+ \\
& +r\left(\frac{\mathrm{d} \boldsymbol{q}_{1}^{(\mathrm{B})}}{\mathrm{d} \psi} \cos \psi+\frac{\mathrm{d} \boldsymbol{q}_{2}^{(\mathrm{B})}}{\mathrm{d} \psi} \sin \psi\right) .
\end{aligned}
$$

The orbital curve on which the object is moving is related to the $\boldsymbol{q}_{j}^{(\mathrm{B})}$-system using the orbit angle $\psi$. Hence its orbital radius reads

$r=r\left(A_{\nu} ; \psi\right)$.

The variation of the orbital radius will be

$\frac{\mathrm{d} r}{\mathrm{~d} \psi}=\frac{\partial r}{\partial \psi}+\sum_{\nu} \frac{\partial r}{\partial A_{\nu}} \frac{\mathrm{d} A_{\nu}}{\mathrm{d} \psi}$.

Therefore, relation (90) leads to

$$
\begin{aligned}
\frac{\mathrm{d} \boldsymbol{r}}{\mathrm{d} \psi}-\frac{\partial \boldsymbol{r}}{\partial \psi}=\left(\sum_{\nu} \frac{\partial r}{\partial A_{\nu}} \frac{\mathrm{dA}_{\nu}}{\mathrm{d} \psi}\right) \boldsymbol{r}_{0}+ \\
r\left(\frac{\mathrm{d} \boldsymbol{q}_{1}^{(\mathrm{B})}}{\mathrm{d} \psi} \cos \psi+\frac{\mathrm{d} \boldsymbol{q}_{2}^{(\mathrm{B})}}{\mathrm{d} \psi} \sin \psi\right) .
\end{aligned}
$$

We now assume the orbit system under consideration to be a Hansen-system with orbit angle

$\psi \triangleq \triangleq=\int\left(G / r^{2}\right) \mathrm{d} t=\int\left|\dot{\boldsymbol{r}}_{0}\right| \mathrm{d} t$ 
In this case, and only in this, because of the Hansen condition (30), the relation

$$
\begin{aligned}
& \frac{\mathrm{d} \boldsymbol{q}_{1}^{(\mathrm{I})}}{\mathrm{d} \zeta} \cos \zeta+\frac{\mathrm{d} \boldsymbol{q}_{2}^{(\mathrm{I})}}{\mathrm{d} \zeta} \sin \zeta= \\
& =\left(\frac{\mathrm{d} \boldsymbol{q}_{1}^{(\mathrm{I})}}{\mathrm{d} t} \cos \zeta+\frac{\mathrm{d} \boldsymbol{q}_{2}^{(\mathrm{I})}}{\mathrm{d} t} \sin \zeta\right) \frac{\mathrm{d} \zeta}{\mathrm{d} t}=0
\end{aligned}
$$

tends to zero in any case. There remains

$\frac{\mathrm{d} \boldsymbol{r}}{\mathrm{d} \zeta}-\frac{\partial \boldsymbol{r}}{\partial \zeta}=\left(\sum_{\nu} \frac{\partial r}{\partial A_{\nu}} \frac{\mathrm{d} A_{\nu}}{\mathrm{d} \zeta}\right) \boldsymbol{r}_{0}$

This behaviour approves Theorem 22: The Lagrange constraint

$\sum_{\nu} \frac{\partial r}{\partial A_{\nu}} \frac{\mathrm{d} A_{\nu}}{\mathrm{d} \zeta}=0$

is necessarily related to a Hansen system using the orbit angle $\zeta$. We conclude: the osculation condition (Lagrange constraint) cannot meaningfully be established without relation to a Hansen system. In this case and only in this case we have

$\frac{\mathrm{d} \boldsymbol{r}}{\mathrm{d} \zeta}=\frac{\partial \boldsymbol{r}}{\partial \zeta}$.

This is a vectorial generalisation of relation (79).

Note: If the Lagrange constraint is not fulfilled, no relation to a Hansen system exists. In this case (and only in this case) the general orbit angle $\psi$ will deviate from the Hansen orbit angle $\zeta$.

\section{Conclusion}

This paper shows that the ideal coordinate system detected by $\mathrm{P}$. Hansen is of central relevance to orbital mechanics. A special insight explains why the method of the variation of parameters works: because any motion is intimately connected with a Hansen system and therefore the variation of parameters will be treated in such a Hansen system.

Although the term "Hansen Ideal" is used in the present paper to refer to Hansen's ideas, it is proposed to use in future only the term "Hansen coordinate system" or simply "Hansen system", as proposed in Brumberg (1995).

Remark: The present paper is devoted to the basic theory only. A series of papers will follow affiliating applications and conclusions.

Acknowledgements. The author thanks cordially Prof. Dr. Bernd Häusler (Universität der Bundeswehr München), Prof. Dr. Otto Wagner (Technical University of Munich), and Prof. Dr. Helmut Süß (German Aerospace Centre, Universität Karlsruhe, Universität der Bundeswehr München) for critical and constructive support for the essential content of this paper. Special thanks should be addressed to Mr. Colin Ward in supporting the accomplishment of the English version of this paper, to M. Sebastien Tailhades and Dipl. Ing. Bastian Calaminus for support in establishing the TeXversion of the paper.

\section{References}

Aiton, E.J.: 1960, Annals of Science 16, 65

Brouwer, D.: 1959a, AJ 64, 378

Brouwer, D.: 1959b,in: G. Birkhoff, R.E. Langer (eds.), Orbit theory, p. 152

Brouwer, D., Clemence, G.M.: 1961, Methods of celestial mechanics, Academic Press, New York

Brown, E.W.: 1896, An introductory treatise on the lunar theory, University Press, Cambridge

Brumberg, V.A.: 1995, Analytical techniques of celestial mechanics, Springer-Verlag, Berlin, Heidelberg, New York

Deprit, A.: 1975, Journal of Research of the National Bureau of Standards - B. Mathematical Sciences. 79B, 1

Deprit, A.: 1976, Celest. Mech. 13, 253

Dziobek, O.: 1962, Mathematical theories of planetary motion, Dover Publications, New York

Efroimsky, M.: 2005, Annals of the New York Academy of Sciences 1065,346

Fisher, D.: 1963, AJ 68, 718

Hansen, P.A.: 1857, Auseinandersetzung einer zweckmäßigen Methode zur Berechnung der absoluten Störungen der Kleinen Planeten, erste Abhandlung, Abhandlungen der mathematisch-physischen Classe der königlich sächsischen Gesellschaft der Wissenschaften, dritter Band, bei S. Hirzel, Leipzig; dritte Abhandlung im fünften Band (1861)

Hansen, P.A.: 1864, Darlegung der theoretischen Berechnung der in den Mondtafeln angewandten Störungen, erste, zweite Abhandlung, Abhandlungen der mathematisch-physischen Classe der königlich sächsischen Gesellschaft der Wissenschaften, sechster Band und siebenter Band (1865), bei S. Hirzel, Leipzig

Herget, P.: 1959, in: G. Birkhoff, R.E. Langer (eds.), Orbit theory, p. 29

Herget, P., Musen, P.: 1958, AJ 63, 430

Jochim, E.F.M.: 2011, Die Verwendung von Hansen-Systemen in Himmelsmechanik und Astrodynamik, DLR-FB-2011-04

Musen, P.: 1958, AJ 63, 426

Musen, P.: 1959, J. Geophys. Res. 64, 2271

Musen, P.: 1961,J. Geophys. Res. 66, 403

Musen, P.: 1963, J. Geophys. Res. 68, 1439

Musen, P.: 1968, Bulletin Astronomique, Serie 3, 3, Fascicule 3, 253

Palacios, M.: 2000, Symplectic versus other propagators in the ideal formulation of the Earth satellite problem', Internat. Symp. Space Dynamics, CNES, June 26-30, 2000, Biarritz

Palacios, M., Calvo, C.: 1996, The Journal of the Astronautical Sciences, 44, 63

Phelan, H.T.: 1962, Computation of satellite orbits by the Hansen Method as modified by Musen, NASA, NASA TR R-147, Goddard Space Flight Center

Struble, R.A.: 1960, Journal of Mathematical Analysis and Applications, 1,300

Struble, R.A.: 1961, Arch. Rational Mech. Anal. 7 , 87

Stumpff, K.: 1974, Himmelsmechanik III, DVW Berlin

Tisserand, F.: 1889-1896, Traité de Mécanique Céleste, Tome I Tome IV, Gauthiers-Villars, Paris

Van der Ha, J.: 1985, Z. Flugwiss. Weltraumforschung 9, 217

Vinh, N.X.: 1970, Celest. Mech. 2, 64

Yarov-Yarovoi, M.S.: 1961, Soviet Ast. 4, 850 